\title{
Cocrystals of betulin with suberic acid: mechanochemical synthesis and thermal behavior
}

\author{
Anastasiya Mikhailovskaya ${ }^{1}$, Svetlana $\mathrm{Myz}^{1}$, Konstantin Gerasimov ${ }^{1}$, Svetlana Kuznetsova ${ }^{2}$, \\ and Tatyana Shakhtshneider ${ }^{{ }^{*}}$ \\ ${ }^{1}$ Institute of Solid State Chemistry and Mechanochemistry SB RAS, Novosibirsk, 630128, Russia \\ ${ }^{2}$ Institute of Chemistry and Chemical Technology, FRC KSC SB RAS, Krasnoyarsk, 660036, Russia
}

\begin{abstract}
In this work, the cocrystals of betulin with suberic acid were prepared by liquid-assisted grinding method using solvents of different polarity. The formation of cocrystals was confirmed by means of powder $\mathrm{X}$-ray diffraction, IR spectroscopy and thermal analysis. It was shown that under heating, a structural transformation occurred after water removal, followed by cocrystal dissociation.
\end{abstract}

\section{Introduction}

Natural products in the roots, stems, barks, leaves, fruits, and seeds of plants have been used as herbal medicines from the ancient times. They also have played a significant role in modern drug discovery by serving as prototypes of novel drugs or the drugs themselves. The interest to natural product-derived drugs flared up with renewed vigor during the coronavirus pandemic $[1,2]$.

Betulin (lup-20(29)-ene-3 $\beta, 28$-diol) is a triterpenoid of lupane-structured pentacyclic triterpene extracted from birch bark. There have been numerous reports on the biological activities of betulin and its derivatives including antiviral, anti-inflammatory, antitumor, and antiprotozoal $[1,2]$. Despite the wide range of biological activity of natural and semisynthetic birch-bark triterpenoids, their studies in vitro and in vivo are rather limited due to the poor solubility and bioavailability. In order to overcome inadequate solubility, various delivery systems and conjugates can be formed such as nanosystems, supramolecular complexes and others [3].

On the other hand, obtaining crystalline forms, for example, cocrystals, is promising for improving the functional properties of the drugs, such as solubility, dissolution rate, stability, etc. [4]. Among many methods of preparing co-crystals, one of the most useful ones is mechanochemical method, especially liquid-assisted grinding (LAG) [5]. In our previous works $[6,7]$, the cocrystals of betulin with dicarboxylic acids, adipic, suberic, and terephthalic acids, were obtained by the LAG method. It has been shown that thus obtained cocrystals possessed higher dissolution rate and solubility compared to the initial betulin.

\footnotetext{
*Corresponding author: shah@,solid.nsc.ru
} 
The purpose of this research was to prepare cocrystals of betulin with suberic acid by mechanochemical methods varying the synthesis conditions, and to study their thermal stability.

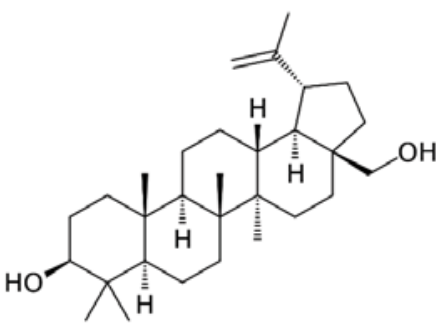

(a)<smiles>O=C(O)CCCCCCC(=O)O</smiles>

(b)

Fig. 1. Molecular structures of betulin (a) and suberic acid (b).

\section{Materials and Methods}

Fig. 1 presents the structural formulas of both betulin and suberic acid. Betulin (BE) was isolated from birch bark according to the developed method [8]. Suberic acid (SubA) (Riedel-de Haen AG, Switzerland) was of analytical grade and was used as received. Ethyl acetate and chloroform (Reachim JSC, Russia) were purified by distillation. Ethanol, acetone, and dioxane (Reachim JSC, Russia, or Sigma-Aldrich, USA) were of analytical grade and were used as received.

To prepare cocrystals, betulin - suberic acid (BE-SubA) mixtures with different molar ratios $(1: 2,1: 1,2: 1)$ were ground in a SPEX 8000 mixer mill (CertiPrep Inc., USA) as it was described in $[6,7]$.

Powder X-ray diffraction (PXRD) analysis was carried out on a D8-Advance diffractometer (Bruker, Germany) with a one-dimensional detector (Lynx Eye type), $\mathrm{CuK}_{\alpha^{-}}$ radiation, $2 \Theta=5-70^{\circ}$. High-temperature measurements were carried out using a hightemperature chamber HTK $1200 \mathrm{~N}$ (Anton Paar, Austria). Powder sample was placed in a corundum carrier and heated in air up to $200{ }^{\circ} \mathrm{C}$ at a heating rate of $0.2{ }^{\circ} \mathrm{C} / \mathrm{s}$. The time of sample keeping at each of the selected temperatures was 10 minutes.

Attenuated total reflectance (ATR) IR spectra were recorded in the $580-4000 \mathrm{~cm}^{-1}$ spectral range using a Digilab Excalibur 3100 FT-IR spectrometer (USA) and a Pike Miracle ATR attachment with ZnSe crystal.

Thermogravimetry (TG) studies were performed using a STA 449 F1 Jupiter (Netzsch, Germany) simultaneous thermal analysis system. The measurements were carried out in $99.990 \%$-pure argon flowing at a volumetric rate of $50 \mathrm{~mL} / \mathrm{min}$. A weighed sample of 30 $50 \mathrm{mg}$ was used for measurements. The heating rate was $10{ }^{\circ} \mathrm{C} / \mathrm{min}$. Calorimetric measurements (DSC) were performed using a DSC 200 F3 MAIA (Netzsch, Germany) heat flux calorimeter. About $5 \mathrm{mg}$ of the solid sample was placed in $40 \mu \mathrm{L}$ closed aluminum caps and scanned from 25 to $250{ }^{\circ} \mathrm{C}$ under argon atmosphere at a heating rate of $6{ }^{\circ} \mathrm{C} / \mathrm{min}$.

\section{Results \& Discussion}

The experiments on liquid-assisted grinding of the mixtures of betulin with suberic acid in various ratios of the initial components showed that the most suitable betulin - suberic acid molar ratio for obtaining a cocrystal was a molar ratio of $1: 1$, with a sufficient time grinding of 20 minutes. In this case, the reflexes of the initial components disappeared completely and new ones appeared, related to the formation of a new crystalline material. 
The use of solvents of different polarity, dioxane, ethanol, acetone, chloroform, and ethyl acetate, led to the same changes in the PXRD patterns (Fig. 2).

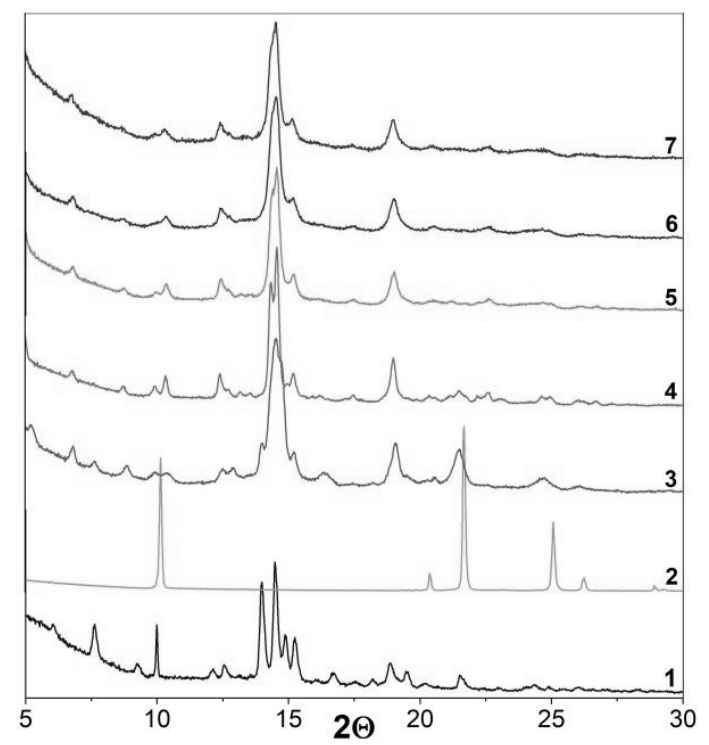

Fig. 2. PXRD patterns of betulin (1), suberic acid (2); 1:1 betulin - suberic acid mixture ground in the presence of ethanol (3), dioxane (4), ethyl acetate (5), acetone (6), and chloroform (7).

Changes in the IR spectra confirmed the formation of a betulin - suberic acid cocrystal after grinding the mixture. Figure 3 shows that as a result of grinding of a 1:1 betulin suberic acid mixture for 20 minutes, the shifts in the $\mathrm{O}-\mathrm{H}$ stretching vibrations (3600-3300 $\mathrm{cm}^{-1}$ ) and C-O-stretching vibrations (about $1020 \mathrm{~cm}^{-1}$ ) of betulin were observed. This suggests that the hydroxyl groups of betulin are involved in intermolecular hydrogen bonds with the carboxyl groups of suberic acid forming cocrystal.
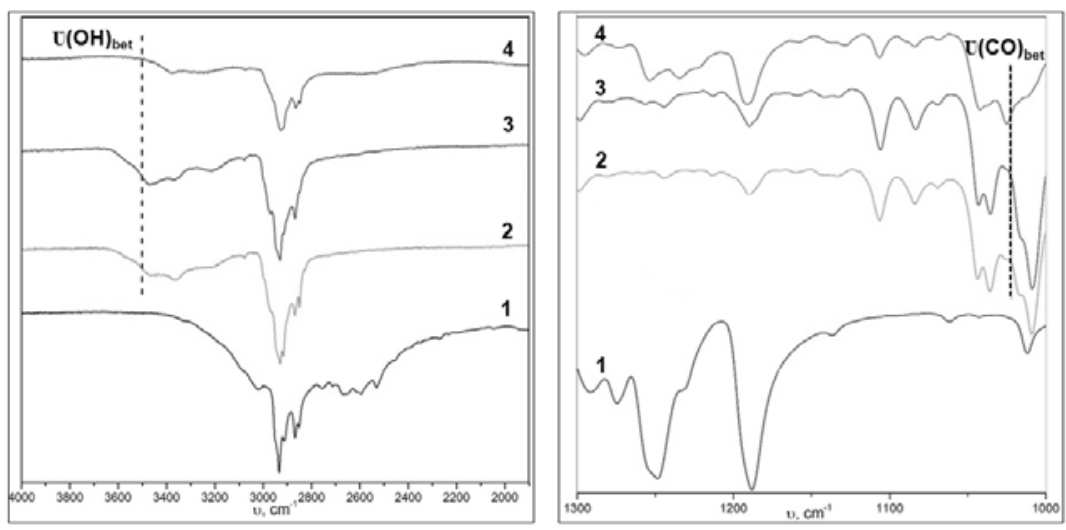

Fig. 3. IR spectra of suberic acid (1); betulin (2), $1: 1$ betulin - suberic acid physical mixture (3); $1: 1$ betulin - suberic acid mixture ground in the presence of dioxane for 20 minutes (4).

On the DSC curve (Fig. 4, a) of the cocrystal obtained in the presence of dioxane, the thermal effects of the initial components $[9,10]$ are absent. There is an endothermic peak in the region of $60-90{ }^{\circ} \mathrm{C}$, which refers to the removal of water. The DSC curve of the cocrystal obtained in the presence of ethyl acetate (Fig. 4, b) shows that similar decomposition processes occur in this sample, except that, apparently, the sample contains 
less water than in the case when dioxane is used as a solvent. Thus, the presence of water in the structure of the co-crystal may be due to the fact that the initial betulin is a betulin hydrate [9], as well as to the presence of water in the liquid used, especially in the case of dioxane due to its hygroscopicity.

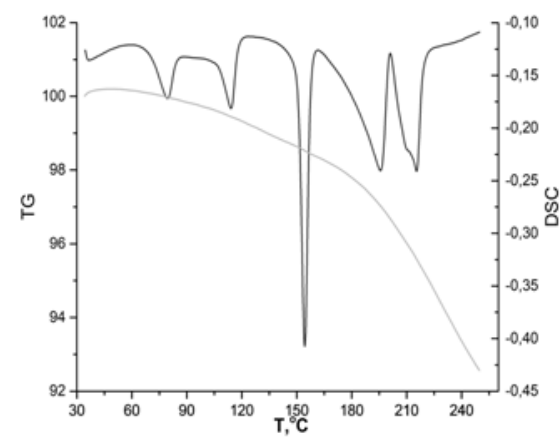

(a)

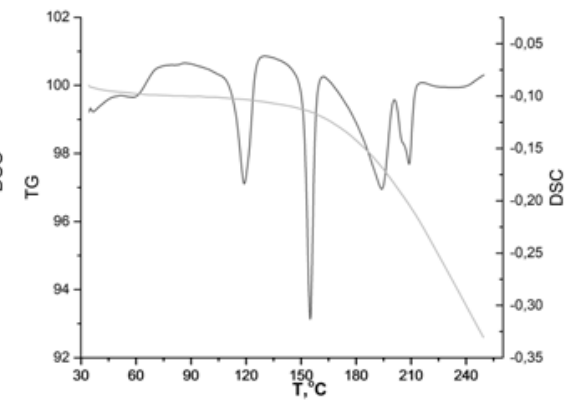

(b)

Fig. 4. TG and DSC curves of BE-SubA cocrystal obtained in the presence of dioxane (a) and ethyl acetate (b).

After removal of water, probably a structural transformation occurs, which corresponds to an endothermic peak in the region of $90-120^{\circ} \mathrm{C}$. This was confirmed by the fact that after drying in a vacuum, the peak of water evolution disappeared, and the peak corresponding to the structural transformation remained on the thermogram. Further, an intense endothermic peak with $\mathrm{Tmax}=155^{\circ} \mathrm{C}$ is observed, in the same temperature range in which a loss of mass proceeded on the TG curve. Presumably, the cocrystal decomposed with the release of gaseous products. Several endothermic peaks in the DSC curve of the cocrystal after $160{ }^{\circ} \mathrm{C}$ could correspond to a further decomposition of the cocrystal and melting of the resulting product. To confirm the interpretation of thermal effects, the PXRD patterns were taken in situ at different temperatures (Fig. 5).

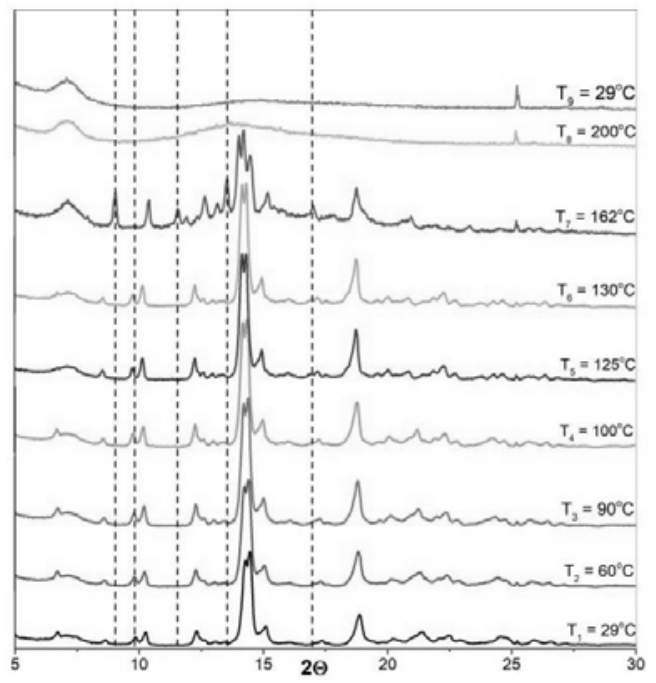

Fig. 5. PXRD patterns of BE-SubA cocrystal taken at different temperatures. 
It is seen from Fig. 6 that no changes in the crystal structure occurred when the cocrystal was heated to $100{ }^{\circ} \mathrm{C}$. This suggests that water loss was not accompanied by a structural transformation of the cocrystal. Near $160{ }^{\circ} \mathrm{C}$, significant changes in the diffraction pattern are observed, which correspond to the decomposition of the cocrystal. When the sample was heated to $200{ }^{\circ} \mathrm{C}$ and above, the melting of the product of cocrystal decomposition was observed.

\section{Conclusion}

Betulin - suberic acid cocrystal was prepared by liquid-assisted grinding method using the solvents of different polarity. As demonstrated by thermal analysis methods and powder Xray diffraction, the cocrystal hydrate was produced during dioxane-assisted grinding. It was shown that betulin - suberic acid cocrystal hydrate was not stable and at heating, transformed at first into the anhydrous form, and then structural transformation proceeded, followed by cocrystal decomposition. The results obtained can be important in the development of dosage forms based on betulin co-crystals.

The work was funded within the framework of the budget projects for the Institute of Solid State Chemistry and Mechanochemistry SB RAS (No. 121032500064-8) and for the Institute of Chemistry and Chemical Technology SB RAS (No. 0287-2021-0017).

\section{References}

1. S. Amiri, S. Dastghaib, M. Ahmadi et al., Biotechnol. Adv. 38, 107409 (2020)

2. M.T. Islam, C. Sarkar, D.M. El-Kersh et al., Phytother. Res. 10, 2471 (2020)

3. I. Mierina, R. Vilskersts, M. Turks, Curr. Med. Chem. 27, 1308 (2020)

4. A.M. Healy, Z.A. Worku, D. Kumar, A.M. Madi, Adv. Drug Deliv. Rev. 117, 25 (2017)

5. D. Hasa, W. Jones, Adv. Drug Deliv. Rev. 117, 147 (2017)

6. S.A. Myz, A.V. Mikhailovskaya, M.A. Mikhailenko et al., Mater. Today: Proc. 12, 82 (2019)

7. A.V. Mikhailovskaya, S.A. Myz, N.V. Bulina et al., Mater. Today: Proc. 25, 381 (2020)

8. S.A. Kuznetsova, G.P. Skvortsova, Ju.N. Malyar et al., Russ. J. Bioorg. Chem. 40, 742 (2014)

9. T.N. Drebushchak, A.V. Mikhailovskaya, V.A. Drebushchak et al. J. Struct. Chem. 61, 1260-1266 (2020)

10. W. Weng, S.N. Wong, X.Y. Xu et al., Cryst. Growth Des. 19, 2736 (2019) 